\title{
Molecular characterization of hepatitis B virus in Vietnam
}

\author{
Thi Ton Taht Bui ${ }^{1}$, Tan Thanh Tran², My Ngoc Nghiem', Pierre Rahman², Thi Thanh Thanh Tran², \\ Man Nguyen Huy Dinh', Manh Hung Le', Van Vinh Chau Nguyen', Guy Thwaites ${ }^{2,4}$ and Motiur Rahman ${ }^{2,4,5^{*}}$ (D)
}

\begin{abstract}
Background: Hepatitis B virus (HBV) infection is a major public health problem globally. HBV genotypes and subgenotypes influence disease transmission, progression, and treatment outcome. A study was conducted among treatment naive chronic HBV patients in southern Vietnam to determine the genotypes and subgenotypes of HBV.
\end{abstract}

Methods: A prospective, exploratory study was conducted among treatment naïve chronic HBV patients attending at the Hospital for Tropical Diseases, in Ho Chi Minh City, Vietnam during 2012, 2014 and 2016. HBV DNA positive samples (systematically selected 2\% of all treatment naïve chronic patients during 2012 and 2014, and $8 \%$ of all treatment naïve chronic patients during 2016) were subjected to whole genome sequencing (WGS) either by Sanger or Illumina sequencing. WGS was used to define genotype, sub-genotype, recombination, and the prevalence of drug resistance and virulence-associated mutations.

Results: One hundred thirty five treatment naïve chronic HBV patients including 18 from 2012, 24 from 2014, and 93 from 2016 were enrolled. Of 135 sequenced viruses, $72.6 \%$ and $27.4 \%$ were genotypes B and C respectively. Among genotype B isolates, $87.8 \%$ and $12.2 \%$ were subgenotypes B4 and B2 respectively. A G1896A mutation in the precore gene was present in $30.6 \%$ of genotype B isolates. The genotype $C$ isolates were all subgenotype $\mathrm{C} 1$ and $78.4 \%$ (29/37) of them had at least one basal core promoter (BCP) mutation. A1762T and G1764 T mutations and a double mutation (A1762T and G1764 T) in the BCP region were significantly more frequent in genotype $C 1$ isolates $(p<0.001)$.

Conclusion: HBV genotype B including subgenotype B4 is predominant in southern Vietnam. However, one fourth of the chronic HBV infections were caused by subgenotype C1.

Keywords: Hepatitis B, Vietnam, Genotype, Subgenotype

\section{Background}

Worldwide, an estimated 2 billion people have been infected with the hepatitis B virus (HBV) and of these 250 million suffer from chronic HBV infection [1].HBV infection either leads to spontaneous recovery or to chronic HBV, which causes chronic liver disease, including liver cirrhosis (LC) and hepatocellular carcinoma (HCC) [2].

\footnotetext{
* Correspondence: mrahman@oucru.org

${ }^{2}$ Oxford University Clinical Research Unit, Toronto, Canada

${ }^{4}$ The Hospital for Tropical Diseases, Wellcome Trust Major Overseas

Programme and Centre for Tropical Medicine, Nuffield Department of

Clinical Medicine, Oxford University, Oxford, UK

Full list of author information is available at the end of the article
}

HBV is a small circular DNA virus $(\sim 3.2 \mathrm{~kb}$ in length) that contains 4 genes with partially overlapping open reading frames (ORFs). These overlapping ORFs encode the polymerase protein, the surface antigen, the core antigen and the $\mathrm{X}$ protein. HBV is highly heterogeneous and is composed of genomes that are closely related but not identical; hence, it is considered as a viral quasispecies within an infected individual [3]. Viral replication is rapid with up to $10^{11}$ virions generated each day in infected individual.Due to the high reverse transcription error rate of the polymerase ( 1 error $/ 10^{7}$ bases) during active infection, $10^{7}$ base-pairing errors can be generated over the 3200-bp genome per day [4].While most of 
these new sequences are within nonviable viruses, they provide a starting point for the emergence of mutants under selective pressure.

HBV can be categorized into 10 different genotypes (A-J; segregated by $<7.5 \%$ genomic sequence diversity) and 40 different subgenotypes (separated by $>4 \%$ genomic sequence diversity) [5]. HBV subgenotyping has caused controversy in the past due to misclassifications and incorrect interpretations from different genotyping methods (whole genome sequence versus $\mathrm{S}$ gene sequence). Criteria for assigning a new subgenotype have been proposed recently [5]. They include: i) analysis of full-length genome, ii) adherence to intra-genotypic nucleotide divergence $(>4.5 \%$ and $<7.5 \%)$, iii) bootstrap values greater than $75 \%$, iv) exclusion of recombinant strains from analysis, v) identification of specific nucleotide and amino acid motifs, vi) a minimum of three purported novel strains, and vii) all available subgenotype strains belonging to the same genotype should be subjected to evolutionary and phylogeny analysis.

HBV genotypes and sub-genotypes differ considerably with respect to geographical distribution, transmission routes, disease progression, responses to antiviral therapy, and clinical outcome, e.g. LC and HCC [6]. The clinical course of infection depends on the host's age at infection, genetic factors, and the genomic variability of the virus, including genotypes, subgenotypes and virulence associated mutations [preS1, preS2, $S$ gene mutations, basal core promoter $(\mathrm{BCP})$, precore $(\mathrm{PC})$ and core mutations [7-9]. Earlier studies have shown that the mutations in the preS 1 and preS2 genes are associated with progression to HCC [9]. The AA positions between 99 to 169 in the $\mathrm{S}$ gene are called the major hydrophobic region (MHR) and the "a" determinant region (aa 124 to 147) is located within the MHR. Mutations in the "a" determinant region cause conformational changes in the $\mathrm{S}$ protein that can affect the antigenicity of $\mathrm{HBsAg}$ and can generate immune escape mutants [10]. Mutations in BCP, PC and core have shown to be associated with HCC [11].

Vietnam is classified as a high burden country regarding hepatitis, and the prevalence of chronic HBV infection is $8-20 \%$ and $31-54 \%$ among the general and the urban high risk populations respectively [12]. Projection and modeling studies have predicted approximately 8 million chronic HBV cases and 58,600 HBV related LC cases in Vietnam by 2025, and that the estimated annual HBV related mortality will be $40,000 /$ year by 2025 [13]. Limited research has been conducted on molecular characterization and determination of virulence associated properties of HBV in Vietnam. Representative data on genotype and subgenotype analysis using whole genome sequence (WGS) [14-16], as well as information on the prevalence of virulence associated mutations in different genes, recombination and drug resistant mutations in treatment naïve patients are limited.

We conducted a prospective exploratory study using systematically and randomly collected HBV isolates from treatment naïve patients attending a tertiary care hospital in southern Vietnam from 2012, 2014 and 2016 respectively. We used WGS to investigate the prevalences of i) genotype and subgenotype, ii) recombinants, iii) primary, secondary and potential nucleos(t)ide analogue (NA) resistance (NAr) mutations, and iv) mutations in the preS1, preS2, S gene, $\mathrm{BCP} / \mathrm{PC}$ and core gene.

\section{Methods}

The study was conducted at the Hospital for Tropical diseases (HTD), Ho Chi Minh City Vietnam from June to December 2012, January to June 2014 and January to December 2016. HTD is a 650 bed tertiary care hospital for infectious diseases and a designated referral center for hepatitis patients for the southern provinces of Vietnam. All treatment naïve chronic HBV patients attending at the hepatitis outpatient department for viral load assays were eligible for the study. Systematically selected residual diagnostic samples from $2 \%$ of the patients (samples from every 50th patient) from 2012 and 2014 and $8 \%$ of the patients from 2016 were included in this study. Serum samples from selected patients were stored at minus $86{ }^{\circ} \mathrm{C}$ until further analysis. Patient address (province, district, city, and wards), clinical chemistry and viral load data were collected from the hospital database. The geolocation of the patients were mapped with QGIS software version 2.18. The study was approved by the Hospital for Tropical Diseases' ethical review committee (Approval No: SC/ND/12/14).

Viral DNA was extracted from $200 \mu \mathrm{L}$ of plasma using QIAamp viral DNA kit (QIAgen GmbH, Hilden, Germany) and eluted in $50 \mu \mathrm{L}$ TBE. The HBV genome was amplified in 4 overlapping fragments (800 bp to $1.2 \mathrm{~kb}$ ) using P1-P2, P3-P4, P5-P6, and P7-P8 primers (P1: 5' -TTT TTC ACC TCT GCC TAA TCA-3'; P2: 5' -TTG GGA TTG AAG TCC CAA TCT GG-3'; P3: 5'-GGG TCA CCT TAT TCT TGG-3'; P4: 5'-ATA ACT GAA AGC CAA ACA GTG GG-3'; P5: 5'-GTC TTC TTG GTT GTT CTT CTA C-3'; P6: 5'-GCA GCA CAG CCT AGC AGC CAT GG-3'; P7: 5'-CCA TAC TGC GGA ACT CCT AGC-3'; P8: 5' -CAA TGC TCA GGA GAC TCT AAG GC-3') [17]. PCR reaction was done in $40 \mu \mathrm{L}$ of buffer containing $50 \mathrm{mM}$ Tris- $\mathrm{HCl}(\mathrm{pH} 8.3), 50 \mathrm{mM}$ $\mathrm{KCl}, \quad 1.5 \mathrm{mM} \mathrm{MgCl} 2,200 \mathrm{mM}$ deoxynucleoside triphosphates (dNTPs), $1 \mathrm{U}$ of Taq DNA-Pwo Polymerase (Expand High Fidelity assay, Boehringer Mannheim), and 30 pmol of primers. The PCR was performed for 35 cycles at $94{ }^{\circ} \mathrm{C}$ for $1 \mathrm{~min}, 58{ }^{\circ} \mathrm{C}$ for $1 \mathrm{~min}$, and $72{ }^{\circ} \mathrm{C}$ for $1 \mathrm{~min}$ in a thermal cycler (ABI 9800) [18]. The PCR products were 
visualized by $1 \%$ agarose electrophoresis and stained with Nancy 520 DNA gel stain. The PCR product was purified using QIAamp PCR product purification kit (QIAgen GmbH, Hilden, Germany). The eluted DNA was quantified by a fluorescence-based dsDNA quantification method using the Quant-iT dsDNA Assay Kit in a Qubit fluorometer (Invitrogen) and was sequenced either by ABI 3100 system after cycle sequencing reaction or by Illumina Myseq system. For the ABI 3100 system, DNA sequencing was done from both ends and consensus sequence was used to construct the whole genome using overlapping fragments. For Illumina sequencing, the amplified fragments were pooled with an equal quantity of each individual PCR amplicon. One nanogram of pooled DNA from individual samples was subjected to library preparation using the Nextera XT DNA sample preparation kit (Illumina, San Diego, CA, USA), in which each sample was assigned to a unique barcode sequence using the Nex-tera XT Index Kit (Illumina). Sequencing of the prepared library was carried out using the Miseq reagent kit v2 (300 cycles, Illumina) in an Illumina Miseq platform.

The Illumina fastq sequence files were assembled using Genious 8.0.5, software package (Biomatters Ltd, AK, New Zeland) utilizing a reference-based mapping tool after primer sequence clipping (i.e. the consensus sequence was obtained by mapping individual reads of each sample to a reference sequence). Finally, screening of minor (sub-consensus) variants was performed using the SNP detection tool available in Geneious. A minimum variant frequency of $5 \%$ and 500 -fold coverage were chosen as cut-off values.

Seventy well characterized HBV WGS representing all genotypes and subgenotypes were downloaded from Gene Bank and the HBV WGS from the current study were subjected to phylogenetic analysis. All complete genome sequences were aligned with MUSCLE from the Genious package. The sequence alignments were then subjected to the Jmodel test to identify the best model for phylogenetic analysis [19]. The suggested nucleotide substitution model $(\mathrm{GTR}+\mathrm{G}+\mathrm{I})$ was subsequently used in phylogenetic analysis using RAxML v7.2.8 (available in the Genious package). To confirm the reliability of phylogenetic tree analysis, bootstrap resampling and reconstruction were carried out 100 times.

All sequences were analyzed for possible recombination by RDP4 v 4.85 software [20]. Any recombination events detected by at least 5 of the 7 programs (RDP, Geneconv, Bootscan, Maxchi, Chimaera, Siscan and Topol) were considered as true recombination. RDP4 v4.85 standard default settings were used, except for Bootscan and Siscan where window sizes of $300 \mathrm{bp}$, step size 30 were used. The prevalence of recombination, recombination breakpoints (start and end point), length of the recombinant fragments and the locations of the recombination were determined.

HBV reverse transcriptase (RT) regions were analyzed for the presence or absence of 42 potential nucleos(t)ide analogue (NA) resistance (NAr) mutations. This includes primary and secondary drug resistance mutations (rt80, rt169, rt173, rt180, rt181, rt184, rt194, rt202, rt204, rt236 and rt250), putative NAar mutations (rt53, rt54, rt82, rt84,rt85, rt91, rt126, rt128, rt139, rt153,rt166, rt191,rt200, rt207,rt213, rt214,rt215,rt217, rt218, rt221, rt229, rt233, rt237, rt238, rt245, and rt256), and pretreatment mutations (rt38, rt124, rt134, rt139, rt224 and rt242) as described earlier [21].

The preS2/S1 sequences were analyzed for preS1 deletion, preS1 mutations (A2962G, C3026A/T, C2964A, and $\mathrm{C} 3116 \mathrm{~T}$ ), preS2 start codon deletion, and preS2 mutations (T31C, T53C, A162G, and T531C/G). The S gene sequence was analyzed for mutations in the "a" determinant region $(\mathrm{T} 116 \mathrm{~N}, \mathrm{P} 120 \mathrm{~S} / \mathrm{T}, \mathrm{I} / \mathrm{T} 126 \mathrm{~S} / \mathrm{A}$, Q129H/R, M133 L/T, K141E, P142S, D144E, and G145R), and other virulence associated mutations (N3S, V184A, and S204R).

Mutations in the BCP (C1653T, T1674C/G, T1753 V, A1762T, G1764/A, C1766T, and T1768A) and the PC/ core region (G1899A, C2002T, A2159G, A2189C, and G2203A/T) associated with HCC were also analyzed.

All data (socio demographic, biochemical and virological) were recorded and analyzed with Statistical Package for the Social Sciences (IBM SPSS version 23, NY, USA). Fisher's exact test was used for the comparison of nominal scale variables and Mann - Whitney U test for ordinal scale variable. A $P$ value $</ 0.05$ was considered to indicate statistically significant difference.

\section{Results}

From June to December 2012, January to June 2014, and June to December 2016 a total of 11,575, 10,329 and 18,141 chronic HBV patients submitted blood samples for HBV viral load assay, and of those 912, 1245 and 1174 patients were treatment naïve respectively. Eighteen samples from 2012 and twenty four from $2014 \quad(2 \%$ of the systematically collected samples) and ninety three samples from 2016 (8\% of the samples) were enrolled in the study.

The geolocations of the 135 patients enrolled in the study were mapped; 87 districts were represented from the 26 southern provinces of Vietnam. Approximately $58 \%$ of the patients were from six provinces including Ho Chi Minh City (28.1\%; 38/135), Dong Nai (6.66\%; 9/135), Long Anh (6.66\%; 9/135), Binh Duong (5.93\%; 8/135), Dong Thap (5.18\%; 7/135) and Tien Giang $(5.18 \%$; $7 / 135)$. The rest of the patients were from 46 districts of the remaining 20 southern provinces of Vietnam (Additional file 1). The gender, 
demographic information, liver enzyme level, viral load and genotype and subgenotype distribution of 135 patients are presented in Table 1 . Approximately half of the patients were male and the mean age of the patients was 32 years. $11.1 \%(15 / 135)$ had a blood ALT concentration of $\geq 5 \mathrm{UNL}$ (reference range is $<37 \mathrm{IU} / \mathrm{l}$ and $<40 \mathrm{IU} / \mathrm{l}$, for females and males, respectively).

The sequence length was $3215 \mathrm{bp}$ for all 135 isolates. Phylogenetic analysis of whole genome sequence showed that the Vietnamese HBV sequences clustered with genotypes $\mathrm{B}$ and $\mathrm{C}$ reference sequences (Fig. 1). Most HBV isolates belong to genotype B (72.6\%; $98 / 135)$, subgenotype B2 (12.2\%; 12/98) and B4 (87.7\%; 86/98). 27.4\% (37/135) of the isolates were genotype $C$ and all genotype $\mathrm{C}$ isolates were belonged to subgenotype $\mathrm{C} 1$. The subgenotype $\mathrm{C} 1$ isolates formed a closely related cluster with a high bootstrapping value (99.99). There were no significant differences in genotypes among i) the isolates from 2012, 2014 and 2016, ii) isolates sequenced by Sanger or Illumina sequencing methods, iii) liver enzyme (AST and ALT) level, iv) viral load and v) geolocation of the patients. All sequences have been deposited in Gen Bank under accession numbers MF621878 and MF674382 - MF674515.

Recombination analysis using RDP identified evidence of recombination in $68.1 \%(92 / 135)$ of the HBV isolates including $93.8 \%(92 / 98)$, and $0.0 \%(0 / 37)$ of genotype $\mathrm{B}, \mathrm{C}$ isolates respectively $(p<0.0001)$. In all recombinant isolates the minor donor was genotype $\mathrm{C}$ including subgenotype $\mathrm{C} 1$. The recombinant sequences were grouped into five groups (Group 1 to Group 5) based on the length of the recombinant fragment. Among the recombinant isolates 14.8\% (20/ 92), $35.5 \%(48 / 92), 5.1 \%(7 / 92), 11.8 \%(16 / 92)$ and 4.4\% (6/92) were recombinant Group 1, 2, 3, 4 and 5 respectively. The recombinant groups, recombinant fragment lengths, recombination breakpoints, and major and minor parents are shown in Fig. 2 and Additional file 2. The majority of the recombination events were identified in the $\mathrm{X}$ gene and in the $\mathrm{PC} / \mathrm{C}$ gene.

Amino acid substitutions at primary and secondary/ compensatory mutations in RT region of polymerase gene were identified in $0.7 \%(1 / 135)$ of the isolates. Putative mutations at NAr were observed in 16.3\%

Table 1 Socio-demographic, biochemical and virological characteristics of 135 patients enrolled in 2102, 2014 and 2016

\begin{tabular}{|c|c|c|c|c|}
\hline \multirow[t]{3}{*}{ Parameter } & All & Genotype B & Genotype C & $p$ value \\
\hline & $\%(n)$ & $\%(n)$ & $\%(n)$ & \\
\hline & $100(135)$ & $72.6(98)$ & $27.4(37)$ & \\
\hline \multicolumn{5}{|l|}{ Subgenotype } \\
\hline B2 & $8.9(12)$ & $12.2(12)$ & & \\
\hline B4 & $63.7(86)$ & $87.7(86)$ & & \\
\hline $\mathrm{C} 1$ & $27.4(37)$ & & $100(37)$ & \\
\hline Age (mean \pm SD) & $33.0 \pm 11.6$ & $31.9 \pm 11.3$ & $35.8 \pm 12.2$ & 0.036 \\
\hline \multicolumn{5}{|l|}{ Sex } \\
\hline Male & $51.9(70)$ & $53.1(52)$ & $48.6(18)$ & $0.395+$ \\
\hline Female & $48.14(65)$ & $46.9(46)$ & $51.4(19)$ & \\
\hline \multicolumn{5}{|l|}{ Year of enrolment } \\
\hline 2012 & $13.3(18)$ & $13.3(13)$ & $13.5(5)$ & \\
\hline 2014 & $17.8(24)$ & $19.4(19)$ & $13.5(5)$ & \\
\hline 2016 & $68.9(93)$ & $67.3(66)$ & $73.3(27)$ & \\
\hline \multicolumn{5}{|c|}{ Biochemistry (median (range) } \\
\hline AST & $10(15.0-707.0)$ & $50.5(15.0-707.0)$ & $53.5(20.0-471.0)$ & $0.509^{*}$ \\
\hline ALT & $69.5(9.0-1025.0)$ & $67.5(9.0-1025.0)$ & $72.5(9.0-594.0)$ & $0.471^{*}$ \\
\hline Creatinine & $83.0(11.0-126.0)$ & $83.0(11.0-114.0)$ & $85.0(45.0-126.0)$ & $0.392^{*}$ \\
\hline Bilirubin & $13.1(6.0-360.0)$ & $13.2(6.0-198.0)$ & $12.8(7.3-360.0)$ & $0.838^{*}$ \\
\hline \multicolumn{5}{|l|}{ HBV Viral load } \\
\hline Mean & $1.45 \times 10^{9}$ & $1.51 \times 10^{9}$ & $1.29 \times 10^{9}$ & \\
\hline (Min-Max) & $2.03 \times 10^{6}-9.35 \times 10^{9}$ & $2.03 \times 10^{6}-6.28 \times 10^{9}$ & $2.65 \times 10^{6}-9.35 \times 10^{9}$ & \\
\hline Median & $8.01 \times 10^{8}$ & $9.29 \times 10^{8}$ & $3.05 \times 10^{8}$ & $0.059^{*}$ \\
\hline
\end{tabular}

†Fisher's exact test; *Mann-Whitney $\mathrm{U}$ test 


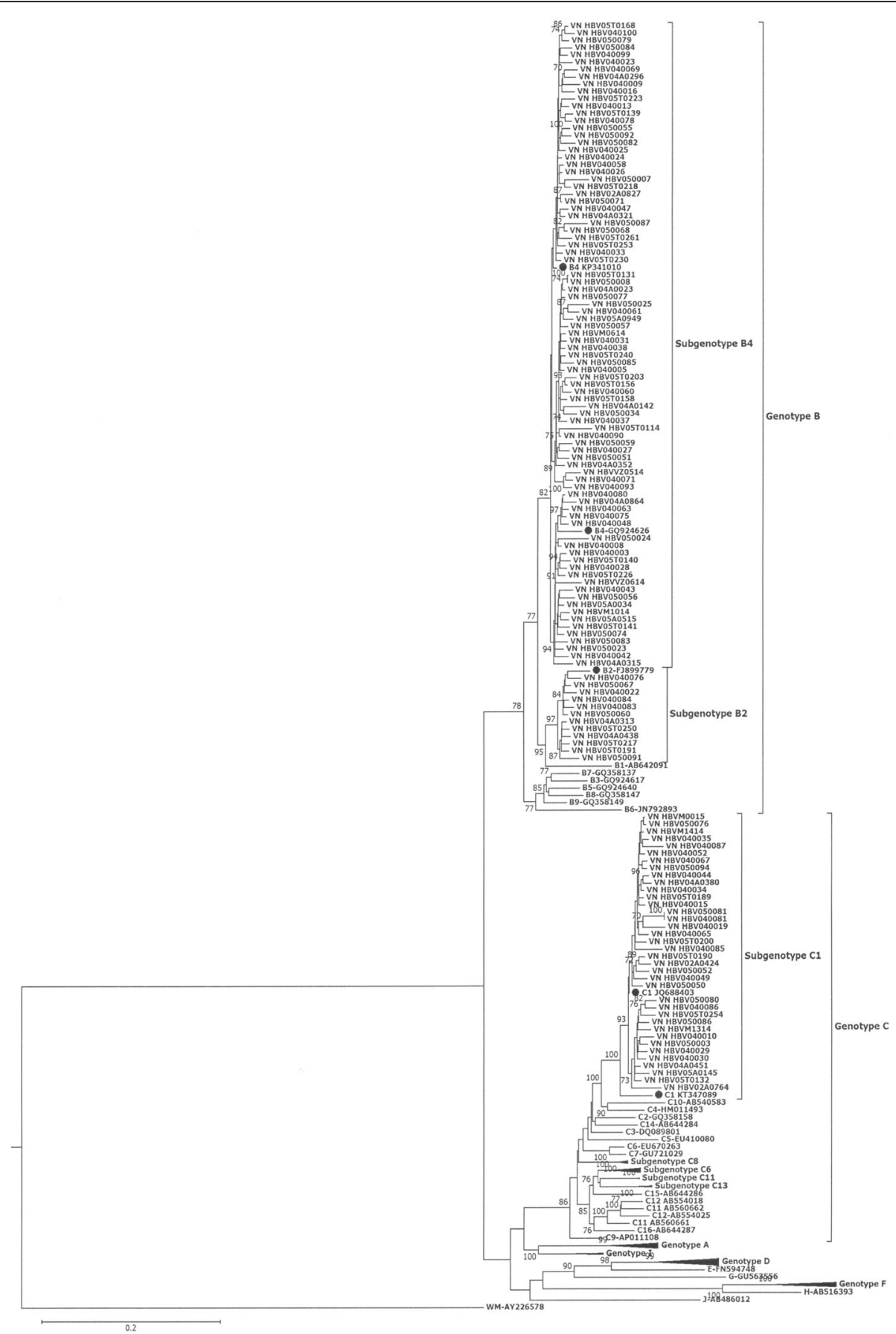

Fig. $1 \mathrm{~A}$ midpoint rooted tree showing the relationship between the Vietnamese HBV genome sequences with 70 reference sequences (all genotypes and subgenotypes). Tree was constructed using RAxML v7.2.8 available in Geneious software using GTR + G + I nucleotide substitution model with 100 bootstrapping replicates. Bootstrap values greater than $70 \%$ are shown on the branch nodes. The Vietnamese HBV strains are presented as VN HBV followed by isolate number and reference genomes are presented as subgenotype followed by Gene Bank accession number. The reference genomes (B2, B4 and (1) are highlighted with a filled dark circle. Reference genomes with multiple sequences for each genotype and subgenotypes (genotype and subgenotype A, D, F, I and subgenotype C6, C8 and C13) were clustered. The scale bar indicates the number of nucleotide substitution. Genotype, subgenotype and Gene Bank accession number of reference sequences are listed in Additional file 3

$(22 / 135)$ isolates including $20.4 \%(20 / 98)$ of the genotype $\mathrm{B}$ and $5.4 \%(2 / 37)$ of the genotype $\mathrm{C}$ isolates $(p=0.026)$. Besides this, we also identified genotype dependant AA polymorphisms at 7 sites. The presence of asparagine or serine at rt53, histidine or tyrosine at rt124, asparagine or aspartic acid at rt134, 


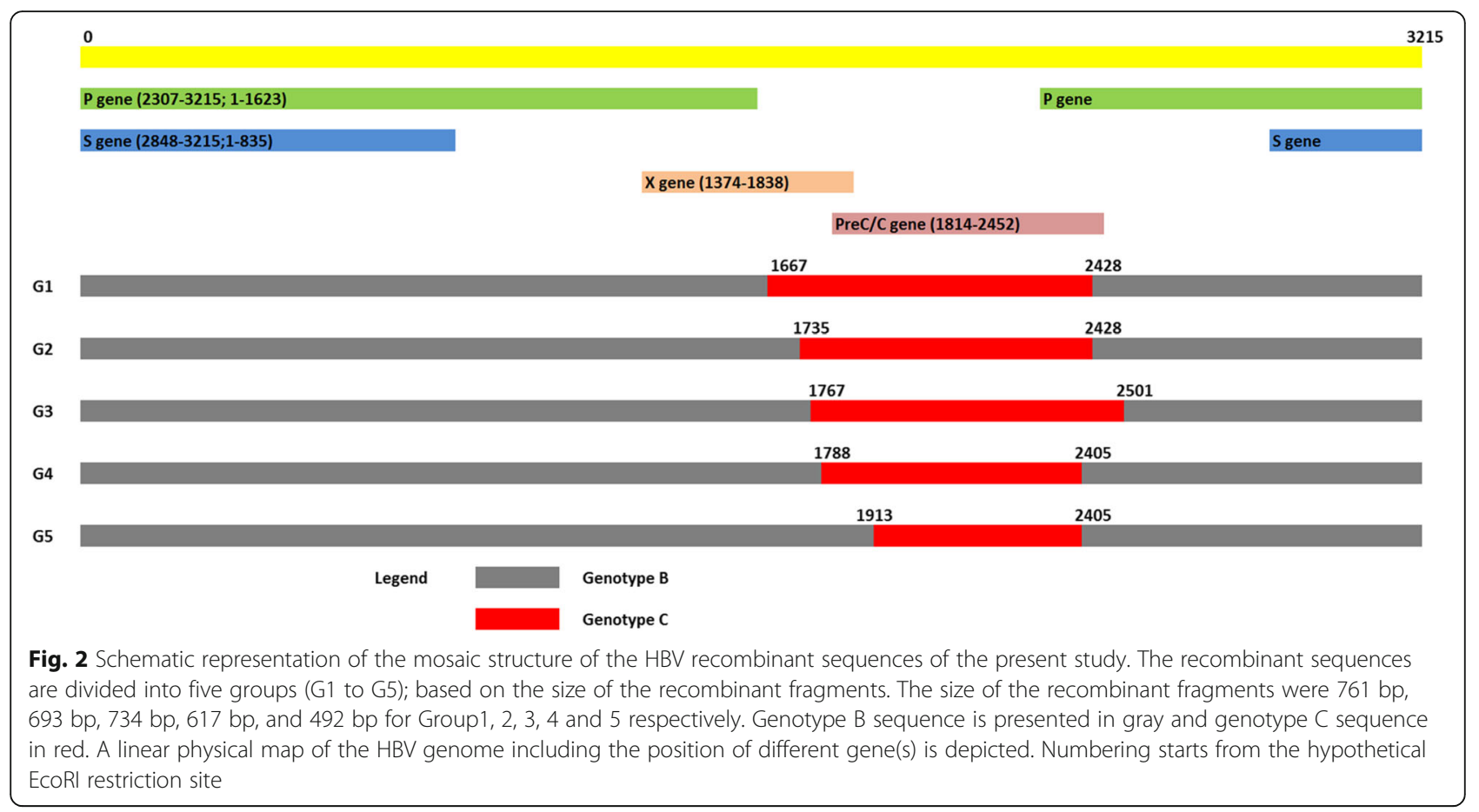

Table 2 Analysis of mutation in the preS1/S2/S gene reported to be associated with HCC and immune escape

\begin{tabular}{|c|c|c|c|c|c|}
\hline Nucleotide & Amino acid & All isolates $N=135 \%(n)$ & Genotype B N = $98 \mathrm{v}$ & Genotype C N = 37\% (n) & $p$ value \\
\hline \multicolumn{6}{|c|}{ Mutation in preS1/S2 and S gene associated with HCC } \\
\hline \multicolumn{6}{|l|}{ PreS1 gene } \\
\hline A2962G & N38E & $71.9(97)$ & $99.0(97)$ & $0.0(0)$ & 0.000 \\
\hline C2964A & N38 K & $71.1(96)$ & $098.0(96)$ & $0.0(0)$ & 0.000 \\
\hline C3026A/T & $\mathrm{A} 60 \mathrm{~V} / \mathrm{E}$ & $100(135)$ & $100(98)$ & $100(37)$ & \\
\hline \multicolumn{6}{|l|}{ PreS2 gene } \\
\hline Start codon & M1 V/T/I & $6.7(9)$ & $8.2(8)$ & $2.1(1)$ & 0.237 \\
\hline T53C & F22 I & $2.2(3)$ & $3.1(3)$ & $0.0(0)$ & 0.379 \\
\hline $\mathrm{A} 162 \mathrm{G}$ & N3S & $27.4(37)$ & $0.0(0)$ & $100(37)$ & 0.000 \\
\hline T531C/G & L126 T/S & $77.0(104)$ & $98.0(96)$ & $21.6(8)$ & 0.000 \\
\hline \multicolumn{6}{|l|}{ S gene } \\
\hline A706C & V184A & $3.7(5)$ & $4.1(4)$ & $2.7(1)$ & 0.581 \\
\hline T766A & S204R & $4.4(6)$ & $6.1(6)$ & $0.0(0)$ & 0.140 \\
\hline \multicolumn{6}{|c|}{ Mutation in $\mathrm{S}$ gene associated with immune escape } \\
\hline \multicolumn{6}{|l|}{ S gene } \\
\hline & P120S/E & $2.2(3)$ & $3.1(3)$ & $0.0(0)$ & 0.379 \\
\hline & I/T126A/N/I/S & $2.2(3)$ & $3.1(3)$ & $0.0(0)$ & 0.379 \\
\hline & M133 L/T & $3.0(4)$ & $4.1(4)$ & $0.0(0)$ & 0.273 \\
\hline & G145R & $0.7(1)$ & $0.0(0)$ & $2.7(1)$ & 0.274 \\
\hline
\end{tabular}


tyrosine or phenylalanine at rt221, valine or isoleucine at rt224, and histidine or asparagine at rt238 were significantly correlated with genotype $\mathrm{B}$ or $\mathrm{C}$, respectively $(p<0.0001)$.Serine at 256 was significantly more associated with genotype $C$ then $B$, though serine was predominant at rt256 for both genotypes.

PreS1/S2/S gene mutation: None of the isolates had preS1 deletions or preS1 start codon mutations. A2962G and C2964A mutations were identified in 99\% (97/98) and $98 \%(96 / 98)$ of the genotype B isolates (Table 2). The preS2 gene start codon mutation $(\mathrm{M} 1 \mathrm{~V} / \mathrm{T} / \mathrm{I})$ was identified in $6.66 \%(9 / 135)$ of the isolates. N3S mutation was significantly higher in genotype $C$ isolates $(p<0.001)$ isolates (Table 2). Among the isolates, $8.1 \%$ $(11 / 135)$ had a mutation in the "a" determinant region including 2.2\% (3/135) P120S/T, 2.2\% (3/135) I/T126S/A, $3 \%(4 / 135) \mathrm{M} 133 \mathrm{~L} / \mathrm{T}$ and $0.7 \%$ (1/135) G145R. There were no significant differences in the mutations between genotype $\mathrm{B}$ and $\mathrm{C}$ isolates (Table 2).

$\mathrm{BCP} / \mathrm{preC} /$ core gene mutation: $\mathrm{BCP}$ mutations at T1753C, G1757A, A1762T, G1764 T and C1766G were analyzed and $30.4 \%(41 / 135)$ of all isolates including $78.4 \%(29 / 37)$ of the $\mathrm{C} 1$ isolates had at least one mutation. T1753C, G1757A, A1762T, G1764A and C1766G mutations were identified in $11.1 \%$ (5/ 135), 7.4\% (10/137), 25.9\%\% (35/135), 24.4\% (33.135) and $2.2 \%(3 / 135)$ of the isolates and A1762T and G1764 $\mathrm{T}$ mutations were significantly more frequent in the genotype $\mathrm{C} 1$ isolates $(p<0.001)$ (Table 3). A double mutation (A1762T and G1764A) was

Table 3 Analysis of mutation in the BCP, precore/core gene reported to be associated with HCC in $135 \mathrm{HBV}$ isolates from southern Vietnam

\begin{tabular}{|c|c|c|c|c|}
\hline Nucleotide & $\begin{array}{l}\text { All isolates } \\
N=135 \%(n)\end{array}$ & $\begin{array}{l}\text { Genotype B } \\
N=98 \%(n)\end{array}$ & $\begin{array}{l}\text { Genotype C } \\
N=37 \%(n)\end{array}$ & $p$ value \\
\hline \multicolumn{5}{|c|}{ Mutation in $\mathrm{BCP}$ region } \\
\hline C1653T & $0.7(1)$ & $1.1(1)$ & $0.0(0)$ & 0.726 \\
\hline T1753C & $11.1(15)$ & $1.1(1)$ & $37.8(14)$ & 0.000 \\
\hline G1757A & $7.4(10)$ & $0(0)$ & $27.0(10)$ & 0.000 \\
\hline A1762T & $25.9(35)$ & $10.2(10)$ & $76.6(25)$ & 0.000 \\
\hline G1764A & $24.4(33)$ & $8.2(8)$ & $76.6(25)$ & 0.000 \\
\hline $\mathrm{A} 1762 \mathrm{~T} / \mathrm{G} 1764 \mathrm{~A}$ & $24.4(33)$ & $8.2(8)$ & $76.6(25)$ & 0.000 \\
\hline C1766G & $2.2(3)$ & $2.0(2)$ & $2.7(1)$ & 0.621 \\
\hline T1768A & $1.4(2)$ & $1.1(1)$ & $2.7(1)$ & 0.475 \\
\hline \multicolumn{5}{|l|}{ Mutation in $\mathrm{PC} /$ Core } \\
\hline G1896A & $22.2(30)$ & $30.6(30)$ & $0(0)$ & 0.000 \\
\hline G1899A & $0.7(1)$ & $1.1(1)$ & $0(0)$ & 0.726 \\
\hline A2159G & $3.7(5)$ & $3.1(3)$ & $5.4(2)$ & 0.419 \\
\hline A2189C & $6.7(9)$ & $6.1(6)$ & $8.1(3)$ & 0.469 \\
\hline G2203A/T & $0.7(1)$ & $1.0(1)$ & $0.0(0)$ & 0.726 \\
\hline
\end{tabular}

identified in $67.5 \%(25 / 37)$ of the $\mathrm{C} 1$ subgenotype isolates compared to $8.2 \%(8 / 98)$ of the genotype B isolates $(p<0.001)$. A G1896A mutation in PC gene was present in $22.2 \%(30 / 135)$ of the isolates, including $30.6 \%(30 / 98)$ of the genotype $\mathrm{B}$ isolates compared to $0 \%$ of the $\mathrm{C} 1$ isolates $(p<0.000)$.

\section{Discussion}

Determination of genotype and subgenotype of HBV is important as they are associated with clinical presentation, transmission, response to therapy and treatment outcome [2]. Considering the large pool of treatment naive chronic HBV patients and limited availability genotyping facilities in Vietnam, genotype and subgenotype data representative of the population are important for clinical decision making, including empirical therapy, disease modeling, and health resource allocation for the management of chronic HBV patients [13]. However, one of the key criteria for assignment of an isolate to a particular subgenotype should be based on analyzing the WGS. In our study we have analyzed the WGS of isolates for genotype and subgenotype determination. We used a 7.5\% diversity across WGS criteria to define an isolate as a particular genotype and $4.5 \%$ to $7.5 \%$ intragenotypic nucleotide divergence for assigning a sequence to a subgenotype [5]. We have collected $2 \%$ and $8 \%$ of the samples of treatment naïve chronic HBV patients attending to a tertiary care and hepatitis referral hospital for southern Vietnam during 2012, 2014, and 2016 in order ensure the representativeness of our data. Besides this, the geolocation analysis indicates that patients enrolled in our study were from 26 provinces including 87 districts of southern Vietnam.

Our data indicate that in southern Vietnam HBV genotype $\mathrm{B}$ is dominant, followed by Genotype C. This is in agreement with earlier data published from Vietnam and southeast Asia using a partial $\mathrm{S}$ gene sequencing approach [15]. Although HBV genotype and subgenotype have distinct geographical distributions, we could not identify such distributions in our study population. This might be due to the fact that both genotype $\mathrm{B}$ and $\mathrm{C}$ is prevalent in southern Vietnam and the subgenotype diversity is limited (i.e. only three subgenotypes are circulating in the southern Vietnam).

Recombination analysis revealed that two thirds of the isolates, including $90 \%$ of the genotype B isolates, are a genotype $\mathrm{B} / \mathrm{C}$ recombinant. This is not surprising as it has been reported that HBV genotype B/Ba (B2-B5) isolates from Vietnam, China, Hong Kong, Indonesia, and Thailand have undergone recombination with $\mathrm{HBV} / \mathrm{C}$ in the core promoter/precore/core genomic region [22]. It is also interesting to note that the HBV genotype B1 isolates (also called $\mathrm{Bj}$ ) from Japan are non recombinant 
and are less virulent compared to HBV genotype B2-B5 (also called $\mathrm{Ba}$ ) isolates from Vietnam, China, Hong Kong, Indonesia and Thailand [23].

We selected patients who were treatment naïve to define the presence of preexisting drug resistance mutations, and to understand the prevalence of virulence associated mutations in the population that are not influenced by treatment selection pressure. With the wide use of NAs, potential NAr mutation positions in $\mathrm{RT}$ region have been reported. We found that less than $1 \%$ of the $\mathrm{HBV}$ isolates had preexisting primary drug resistance mutations. We have identified NAr resistant mutations in $16.4 \%$ of the isolates, which are similar to earlier data from the region where $25 \%$ of the isolates had such mutations [24]. In this study seven genotype-dependent AA polymorphic positions were identified for B- and C- genotypes. This is in agreement with earlier reports from China; however we could not identify genotype-dependent AA polymorphic positions at the rt91 position. This might be due to evolution and adaption of HBV isolates in the Vietnamese population.

None of the isolates in our study had a preS deletion which is associated with higher risk for HCC. However, longitudinal studies showed that the preS deletion mutations occur during the long course of liver diseases, but not at the beginning of HBV infection [7]. As patients selected in our study are treatment naïve, it may be that they have been infected only for a relatively short time. It is interesting to note that $8.1 \%$ of the isolates had mutations in the "a" determinant region. These mutations can affect the antigenicity of HBsAg and have shown to be responsible for: i) false-negative results by commercial assays for HBsAg; ii) evasion of anti-HBV immunoglobulin therapy, and iii) evasion of vaccine induced immunity. These "vaccine-escape" mutants are more common in countries with high rates of endemic infections and universal immunization programs [25]. Further studies on the association between mutations in the "a" determinant region and vaccination status of the patients in Vietnam would provide insight in the role of these mutations.

HBV genotype $C$ is known to be more virulent and associated with poor clinical outcome often leading to HCC. Genotype C isolates in our study belonged to subgenotype $\mathrm{C} 1$ and were tightly clustered with high prevalence of known virulence mutations (e.g. A1762T \& G1764 $\mathrm{T}$ double mutation) [15]. Approximately one quarter of the genotype B patients had a G1896A mutation. This mutation creates a premature stop codon in the $P C$ gene, prevents translation of $P C$ protein, and abolishes the production of $\mathrm{HBeAg}$ and results in HBeAg seroconversion. This is in agreement with earlier reports indicating a low $\mathrm{HBeAg}$ positivity and earlier HBeAg seroconversion in genotype B isolates [26].

Data on transmission of $\mathrm{HBV}$ are lacking in Vietnam. However, perinatal/vertical transmission is reportedly more common in genotype $\mathrm{B}$ and $\mathrm{C}$ compared to A, D and E-J [26]. Taken together our data suggest that along with the predominant HBV subgenotype B4, subgenotype $\mathrm{C} 1$ variant is also circulating in southern Vietnam and of public health significance.

A limitation of our study was the lack of clinical data on the patients. Such data would support the clinical relevance of the genotype and subgenotype. Longitudinal studies on patients with specific genotype and subgenotype infection are essential to fill this knowledge gap.

\section{Conclusions}

HBV genotype B and C predominates in Vietnam. Among genotype $\mathrm{B}$ and $\mathrm{C}$ isolates, $\mathrm{B} 4$ and $\mathrm{C} 1$ subgenotype are predominating respectively. Subgenotype $\mathrm{C} 1$ isolates contains significantly more virulence associated mutations compared to genotype B isolates.

\section{Additional files}

\begin{abstract}
Additional file 1: Geolocation mapping of the 135 chronic HBV patients enrolled in the study. Description: Addresses of each enrolled patient were mapped by QGIS v2.18 at ward level. Geolocation of wards of genotype $B$ patients are marked red, genotype $C$ patients as blue and wards with patients of both genotype $B$ and $C$ are marked as green (DOCX 319 kb)

Additional file 2: Recombination analysis. Recombination analysis of the $\mathrm{HBV}$ isolates from this study using RDP4 $\vee 4.85$ program. Recombination analysis of the $92 \mathrm{HBV}$ isolates from this study using RDP4 $\vee 4.85$ program. An isolate was considered recombinant if detected by 5 out of 6 program (RDP, BootScan, Max Chi, Chimaera, SisScan and Topol). Recombinant isolate, minor parents and identity, recombinant break points, size of the recombinant fragment and location of the recombination are presented. (PPTX $141 \mathrm{~kb}$ )

Additional file 3: HBV reference isolates_180817. The HBV reference genome sequence and sequences isolated from Vietnam in this study used for phylogentic analysis. Gene Bank accession number and subgenotype of the reference sequences used for phylogenetic analysis. Gene Bank accession number and subgenotype of the HBV isolates from this study. (DOCX $12 \mathrm{~kb}$ )
\end{abstract}

\section{Abbreviations}

AA: Amino acid; ALT: Alanine aminotransferase; AST: Aspartate aminotransferase; BCP: Basal core promoter; DNA: Deoxyribonucleic acid; dNTPs: Deoxynucleoside triphosphates (dNTPs); HBV: Hepatitis B virus; HCC: Hepatocellular carcinoma; HTD: Hospital for Tropical Diseases; IU: International Unit; LC: Liver cirrhosis; NA: Nucleos(t)ide analogue; NAr: Nucleos(t)ide analogue resistance; ORFs: Open reading frames; PC: Pre core; PCR: Polymerase chain reaction; RT: Reverse transcriptase; SNP: Single nucleotide polymorphism; UNL: Upper Normal Limits; WGS: Whole genome sequencing

\section{Acknowledgements}

We thank the Li Ka Shing foundation for funding the work. We thank Drs. Stephen Baker, Rogier van Doorn, Maciek Boni, Maia Rabaa Jeremy Day for their valuable comments on phylogenetic analysis. 


\section{Authors' contribution}

TBTT, Sample collection, performing sequencing work, data compilation and analysis, manuscript writing and review. PR, Sequencing work, data compilation and analysis, manuscript writing and review. TT, Data analysis, manuscript writing and review. NNM, Study design, proposal writing, study supervision, manuscript writing and review. TTाT, Geolocation mapping of patients' data, mutation analysis, review of manuscript. MDNH, Study design, proposal writing, study supervision, manuscript review. HLM, Study design, proposal writing, analysis plan, study supervision, manuscript writing and review. NWC, Study design, analysis plan, study supervision, manuscript writing and review. GT, Study design, study supervision, manuscript writing and review. MR, Study design, proposal writing, analysis plan, study supervision, manuscript drafting. All authors read and approved the final manuscript.

\section{Funding}

The study was funded by "Li Ka Shing foundation" fund to Oxford University.

\section{Availability of data and materials}

The WGS of the $135 \mathrm{HBV}$ isolates is submitted to Gene bank. The accession number is MF621878 and MF674382 - MF674515.

\section{Ethics approval and consent to participate}

The study was approved by the Hospital for Tropical Diseases' ethical review committee. The approval number is SC/ND/12/14.

\section{Consent for publication}

Not applicable.

\section{Competing interests}

The authors declare that they have no competing interests.

\section{Publisher's Note}

Springer Nature remains neutral with regard to jurisdictional claims in published maps and institutional affiliations.

\section{Author details}

${ }^{1}$ Hospital for Tropical Diseases, Ho Chi Minh City, Vietnam. ${ }^{2}$ Oxford University Clinical Research Unit, Toronto, Canada. ${ }^{3}$ Mount Sinai Hospital, Toronto, Canada. ${ }^{4}$ The Hospital for Tropical Diseases, Wellcome Trust Major Overseas Programme and Centre for Tropical Medicine, Nuffield Department of Clinical Medicine, Oxford University, Oxford, UK. ${ }^{5}$ Laboratories, Centre for Tropical Medicine, Oxford University Clinical Research Unit, 764 Vo Van Kiet Street, Ward 1, District 5, Ho Chi Minh City, Vietnam.

\section{Received: 8 December 2016 Accepted: 21 August 2017}

\section{Published online: 31 August 2017}

\section{References}

1. Schweitzer A, Horn J, Mikolajczyk RT, Krause G, Ott JJ. Estimations of worldwide prevalence of chronic hepatitis B virus infection: a systematic review of data published between 1965 and 2013. Lancet. 2015;386(10003):1546-55.

2. Te HS, Jensen DM. Epidemiology of hepatitis B and C viruses: a global overview. Clin Liver Dis. 2010;14(1):1-21. vii

3. Ngui SL, Hallet R, Teo CG. Natural and iatrogenic variation in hepatitis B virus. Rev Med Virol. 1999;9(3):183-209.

4. Locarnini S, Hepatitis B. viral resistance: mechanisms and diagnosis. J Hepatol. 2003;Suppl 1:S124-32.

5. Pourkarim MR, Amini-Bavil-Olyaee S, Kurbanov F, Van Ranst M, Tacke F. Molecular identification of hepatitis B virus genotypes/subgenotypes: revised classification hurdles and updated resolutions. World J Gastroenterol. 2014;20(23):7152-68.

6. Tanwar S, Dusheiko G. Is there any value to hepatitis B virus genotype analysis? Curr Gastroenterol. 2012;14(1):37-46.

7. Qu LS, Liu JX, Liu TT, Shen XZ, Chen TY, Ni ZP, Lu CH. Association of hepatitis B virus pre-S deletions with the development of hepatocellular carcinoma in Qidong, China. PLoS One. 2014;9(5):e98257.

8. Zhang $X$, Ding HG. Key role of hepatitis B virus mutation in chronic hepatitis B development to hepatocellular carcinoma. World J Hepatol. 2015;7(9):1282-6.
9. Yano Y, Azuma T, Hayashi Y. Variations and mutations in the hepatitis B virus genome and their associations with clinical characteristics. World Hepatol. 2015;7(3):583-92.

10. Caligiuri P, Cerruti R, Icardi G, Bruzzone B. Overview of hepatitis B virus mutations and their implications in the management of infection. World $J$ Gastroenterol. 2016;22(1):145-54.

11. Tong MJ, Blatt LM, Kao JH, Cheng JT, Corey WG. Precore/basal core promoter mutants and hepatitis B viral DNA levels as predictors for liver deaths and hepatocellular carcinoma. World I Gastroenterol. 2006;12(41):6620-6.

12. Gish RG, Bui TD, Nguyen CT, Nguyen DT, Tran HV, Tran DM, Trinh HN. International Group for Liver Health in Viet N. Liver disease in Viet Nam: screening, surveillance, management and education: a 5-year plan and call to action. J Gastroenterol Hepatol. 2012;27(2):238-47.

13. Nguyen VT, Law MG, Dore GJ. An enormous hepatitis B virus-related liver disease burden projected in Vietnam by 2025. Liver Int. 2008;28(4):525-31.

14. Tran HT, Ushijima H, Quang VX, Phuong N, Li TC, Hayashi S, Xuan Lien T, Sata T, Abe K. Prevalence of hepatitis virus types B through $E$ and genotypic distribution of HBV and HCV in Ho Chi Minh City, Vietnam. Hepatol Res. 2003;26(4):275-80.

15. Huy TT, Ushijima H, Quang VX, Ngoc TT, Hayashi S, Sata T, Abe K. Characteristics of core promoter and precore stop codon mutants of hepatitis B virus in Vietnam. J Med Virol. 2004;74(2):228-36.

16. Thuy le TT, Ryo H, Van Phung L, Furitsu K, Nomura T. Distribution of genotype/subtype and mutational spectra of the surface gene of hepatitis $B$ virus circulating in Hanoi, Vietnam. J Med Virol. 2005;76(2):161-9.

17. Friedt $M$, Gerner P, Wintermeyer P, Wirth S. Complete hepatitis B virus genome analysis in $\mathrm{HBsAg}$ positive mothers and their infants with fulminant hepatitis B. BMC Gastroenterol. 2004;4:11.

18. Shi W, Zhu C, Zheng W, Zheng W, Ling C, Carr MJ, Higgins DG, Zhang Z. Subgenotyping of genotype $C$ hepatitis B virus: correcting misclassifications and identifying a novel subgenotype. PLoS One. 2012;7(10):e47271.

19. Posada D. jModelTest: phylogenetic model averaging. Mol Biol Evol. 2008;25(7):1253-6.

20. Martin DP, Murrell B, Golden M, Khoosal A, Muhire B. RDP4. Detection and analysis of recombination patterns in virus genomes. Virus Evol. 2015;1(1):vev003.

21. Liu BM, Li T, XU J, Li XG, Dong JP, Yan P, Yang JX, Yan L, Gao ZY, Li WP, et al. Characterization of potential antiviral resistance mutations in hepatitis $B$ virus reverse transcriptase sequences in treatment-naive Chinese patients. Antivir Res. 2010;85(3):512-9.

22. Sugauchi F, Orito E, Ichida T, Kato H, Sakugawa H, Kakumu S, Ishida T, Chutaputti A, Lai CL, Ueda R, et al. Hepatitis B virus of genotype B with or without recombination with genotype $\mathrm{C}$ over the precore region plus the core gene. J Virol. 2002;76(12):5985-92.

23. Sakamoto T, Tanaka Y, Simonetti J, Osiowy C, Borresen ML, Koch A, Kurbanov F, Sugiyama M, Minuk GY, McMahon BJ, et al. Classification of hepatitis B virus genotype B into 2 major types based on characterization of a novel subgenotype in Arctic indigenous populations. J Infect Dis. 2007;196(10):1487-92.

24. Xu J, Wu B, Wang JH, Huang L, Wang DY, Zhao L, Zhao GP, Wang Y. Pre-existing mutations in reverse transcriptase of hepatitis $B$ virus in treatment-naive Chinese patients with chronic hepatitis B. PLoS One. 2015;10(3):e0117429.

25. Lazarevic I. Clinical implications of hepatitis B virus mutations: recent advances. World J Gastroenterol. 2014;20(24):7653-64.

26. Sunbul M. Hepatitis B virus genotypes: global distribution and clinical importance. World J Gastroenterol. 2014;20(18):5427-34. 